Gary Sean Cooney*, Manuel Barberio, Michele Diana, Robert Sucher, Claire Chalopin and Hannes Köhler

\title{
Comparison of spectral characteristics in human and pig biliary system with hyperspectral imaging (HSI)
}

https://doi.org/10.1515/cdbme-2020-0012

\begin{abstract}
Injuries to the biliary tree during surgical, endoscopic or invasive radiological diagnostic or therapeutic procedures involving the pancreas, liver or organs of the upper gastrointestinal tract give rise to the need to develop a method for clear discrimination of biliary anatomy from surrounding tissue. Hyperspectral imaging (HSI) is an emerging optical technique in disease diagnosis and image-guided surgery with inherent advantages of being a non-contact, non-invasive, and non-ionizing technique. HSI can produce quantitative diagnostic information about tissue pathology, morphology, and chemical composition. HSI was applied in human liver transplantation and compared to porcine model operations to assess the capability of discriminating biliary anatomy from surrounding biological tissue. Absorbance spectra measured from bile ducts, gall bladder, and liver show a dependence on tissue composition and bile concentration, with agreement between human and porcine datasets. The bile pigment biliverdin and structural proteins collagen and elastin were identified as contributors to the bile duct and gall bladder absorbance spectra.
\end{abstract}

Keywords: bile; biliary tree; hyperspectral imaging; imageguided surgery; visualization.

*Corresponding author: Gary Sean Cooney, Innovation Center for Computer Assisted Surgery (ICCAS), Universität Leipzig, Leipzig, Germany, E-mail: Gary.Cooney@medizin.uni-leipzig.de Manuel Barberio and Michele Diana, IHU-Strasbourg Institute of Image-Guided Surgery, Strasbourg, France

Robert Sucher, Department of Visceral, Transplant, Thoracic and Vascular Surgery, University Clinic Leipzig, Leipzig, Germany Claire Chalopin and Hannes Köhler, Innovation Center for Computer Assisted Surgery (ICCAS), Universität Leipzig, Leipzig, Germany

\section{Introduction}

\section{Biliary injuries and HSI}

Iatrogenic biliary injuries caused by surgical, endoscopic, diagnostic, or therapeutic procedures of the upper gastrointestinal system can severely impact post-operative quality of life and mortality [1]. Common causes of injuries include the visual misperception and differences in biliary anatomy from patient to patient [2]. Traditional intraoperative cholangiography (IOC) requires the use of ionizing radiation and an intraoperative C-Arm. This disrupts surgical workflow and exposes patients and staff to radiation. While there have been recent advancements in biliary visualization methods such as fluorescent IOC [3], these methods still require the administration of exogenous fluorescent dyes.

Hyperspectral imaging (HSI) is an emerging biomedical imaging technique that produces both spatial and spectral information by combining standard imaging with visible and near-infrared (VNIR) spectroscopy. HSI allows for the noncontact and non-invasive characterization of tissue morphology, composition and physiology using endogenous chromophores present in biological tissue [4]. Tissue parameters such as tissue oxygenation saturation $\left(\mathrm{StO}_{2}\right)$ and tissue perfusion can therefore be calculated and interpreted for the diagnosis of physiology and pathology. HSI has been previously shown to give reliable results in visceral surgery (intestinal resections [5, 6], left liver resections [7, 8], esophagectomy [9]), with various case studies currently in progress.

In this study, HSI data from human liver transplantation procedures and porcine model operations were studied and compared to discriminate biliary structures from surrounding tissue. Chromophores present in bile fluid and tissue composition were explored as cause of characteristic absorbances observed in recorded HSI spectra of liver, gall bladder, and bile ducts.

\section{Bile and biliary tissue composition}

Bile is an alkaline aqueous liquid produced continuously by the liver and is stored and concentrated in the gall bladder. 
The stored bile is discharged into the duodenum via the common bile duct after food intake to aid in the digestion of lipids. Bile mainly constitutes of water $(\sim 95 \%)$ with the remaining $5 \%$ consisting of various organic and inorganic solutes such as bile salts, cholesterol, immune cells, hormones, and bicarbonate $\left(\mathrm{HCO}_{3}^{-}\right)$[10]. However, primary bile salts cholic acid (CA) and chenodeoxycholic acid (CDCA), as well as other small molecules present such as cholesterol and glutathione absorb light within the UV-range $(<400 \mathrm{~nm})$. This is not detectable to HSI operating within the VNIR window (500-1,000 nm). Bile is the major route of excretion of bilirubin [10] which along with the intermediate compound biliverdin, are catabolic products of heme from the breakdown of red blood cells in the liver. Bilirubin and biliverdin absorb light at 460 and $675 \mathrm{~nm}$ respectively which, although present in small quantities in bile, contributes to its yellow-green appearance. These bile pigments mainly exist in a water-soluble conjugated state which can cause a shift in the observed absorbance [11]. Moreover, the absorption coefficient of biliverdin at $675 \mathrm{~nm}$ is greater than oxygenated hemoglobin $\left(\mathrm{HbO}_{2}\right)$ [12].

The gall bladder contains contractile tissue for the release of bile into the ileum upon signaling, which constitute of the structure proteins collagen and elastin. This contractility differs from the main connective tissue of the liver, Glisson's Capsule which surrounds the liver and hepatic ducts, arteries, and veins [13]. Both collagen and elastin have broad absorbances in the $500-850 \mathrm{~nm}$ range, with local maxima centered around $910 \mathrm{~nm}$ [14].

\section{Materials and methods}

\section{Liver transplantation data}

During liver transplantation procedures (before and after allograft) of $\mathrm{n}=7$ patients ( 4 male, 3 female) 22 intraoperative hyperspectral images were acquired with the TIVITA ${ }^{\circ}$ Tissue system (Diaspective Vision $\mathrm{GmbH}$, Am Salzhaff, Germany). This HSI push-broom scanner operates in the visible and near-infrared range (500-1000 nm), having a high spectral resolution $(5 \mathrm{~nm})$ with $640 \times 480$ effective pixels $(x-, y$-axis). A distance of $50 \mathrm{~cm}$ between the object and camera was maintained giving a focal length of $25 \mathrm{~mm}$ and an $8 \times 6.5 \mathrm{~cm}^{2}$ field of view (FOV). Object illumination is performed by six halogen spots ( $20 \mathrm{~W}$ each) which are integrated into the camera housing. To reduce background light interference, ceiling lights were switched off during imaging recording which takes about $8 \mathrm{~s}$ to produce the hyperspectral data cube.

\section{Pig model data}

Five intraoperative in vivo hyperspectral images of porcine upper abdomen, including bile duct, liver and gallbladder were similarly acquired using the TIVITA ${ }^{\circledR}$ Tissue system.

\section{Data processing}

The location of gall bladder, biliary ducts, liver, peritoneum, and surrounding connective tissues were annotated on the color images by the operating physician which in turn, were used to generate region of interests (ROIs). The data was pre-processed using Microsoft Excel and MATLAB. The Standard Normal Variate (SNV) transformed spectra of ROI were comparted initially for the identification of visible outliers. SNV transformation normalizes a set of spectra and removes the baseline effect, effectively minimizing scatter effects while accentuating spectral features. Outliers include incorrectly annotated tissue, spectra affected by glare or movement artefacts.

After outlier removal, the raw ROI spectra were averaged across all included patients to give average absorption spectra for each of the tissue classes. Subsequently, derivative spectra using a SavitzkyGolay filter (polynomial order $=2$, window length $=5$ ) were produced to reduce the influence of broad absorbance bands resulting from scattering effects.

\section{Results}

The averaged absorbance spectra (Figure 1) shows the liver has the highest absolute absorbance in both human and pig datasets. This is evident within the $500-700 \mathrm{~nm}$ range, which can be attributed to the high blood concentration of both oxygenated hemoglobin, $\mathrm{HbO}_{2}$ (550 and $600 \mathrm{~nm}$ ) and deoxygenated, $\mathrm{HHb}(650 \mathrm{~nm})$ [12].

Both human and pig bile ducts have a similar spectral profile, having weaker relative absorption than liver and gall bladder between 600 and 1,000 $\mathrm{nm}$. This could be due to the lower tissue and bile volume relative to the liver and gall bladder, as well as overlying tissue layers causing greater scattering and thus attenuating the observed absorbance signal. Human and pig gall bladder spectra have a distinct broad absorbance from $600 \mathrm{~nm}$ onwards. This reflects the higher bile content expected in the gall bladder and suggests a distinct tissue composition from that of the bile ducts.

The second-order derivative spectra (Figure 2) results in observable negative absorption peaks with minima at the same wavelength as the maxima on the zero-order absorption spectrum. Five distinct bands were identified in the region 645-1,000 $\mathrm{nm}$ corresponding to biliverdin, deoxyand oxy-hemoglobin $\left(\mathrm{HHb}\right.$ and $\left.\mathrm{HbO}_{2}\right)$, lipid and water.

\section{Discussion}

The intrahepatic biliary tract is unlikely to be distinguishable given the absorption of the liver (red) is greater than bile ducts (green) at any given wavelength. An exception to this is evident in the human data beyond $930 \mathrm{~nm}$, where an 

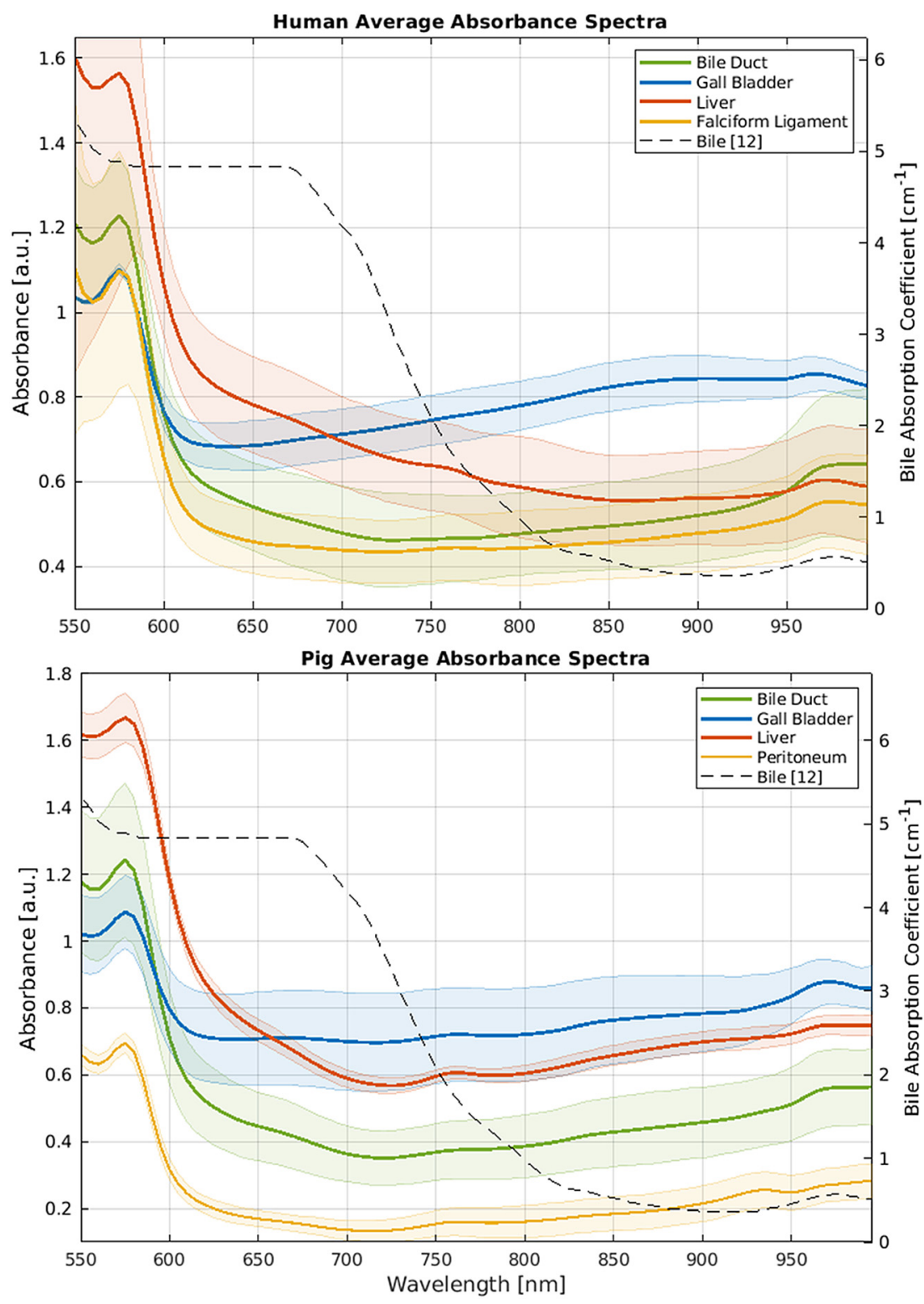

Figure 1: Averaged absorbance spectra of human (top) and porcine (bottom) tissue with standard deviations as shaded area and bile absorption coefficient [12] plotted as secondary $y$-axis. increased absorbance in the bile duct is observed. This absorbance is hypothesized to be caused by the high-water content of bile ( $95 \%)$ as well as the high-lipid content [10], which have expected maxima of ca. 970 and $930 \mathrm{~nm}$ respectively [15].

Bile is believed to be a cause of the characteristic broad absorbance seen in liver, gall bladder, and bile duct spectra. The reported bile absorption coefficient [12] (secondary $y$ axis) features a local maximum at $675 \mathrm{~nm}$ associated with bile pigment biliverdin. This indicates bile pigments having a greater contribution to the absorption of bile-containing tissue spectra than $\mathrm{HbO}_{2}$ at equal concentrations.

While bile on average is alkaline, it has been shown that the bile contained in the gall bladder becomes increasingly more acidic during fasting periods [16]. In general, gall bladder bile tends to be of a lower $\mathrm{pH}$ (6.807.65), compared that of the common bile duct ( $\mathrm{pH}=7.50-$ 8.05). These $\mathrm{pH}$ variations influence the composition of bile, especially in the formation of conjugates [17]. It has also been demonstrated that at low $\mathrm{pH}<4$ oxidation of bilirubin to biliverdin takes place in pure bile, leading to an increased absorbance of the latter at ca. $700 \mathrm{~nm}$. It is therefore possible that perioperative fasting can lead to a decreased bile $\mathrm{pH}$ and increase in biliverdin concentration, which is to be considered when using biliverdin in future biliary classification algorithms. This broad absorbance is evident in both human and pig gall bladder spectra in the range $550-750 \mathrm{~nm}$. However, the proposed contribution of biliverdin to the spectra of liver and gall bladder does not explain the difference observed between 700 and $950 \mathrm{~nm}$. 

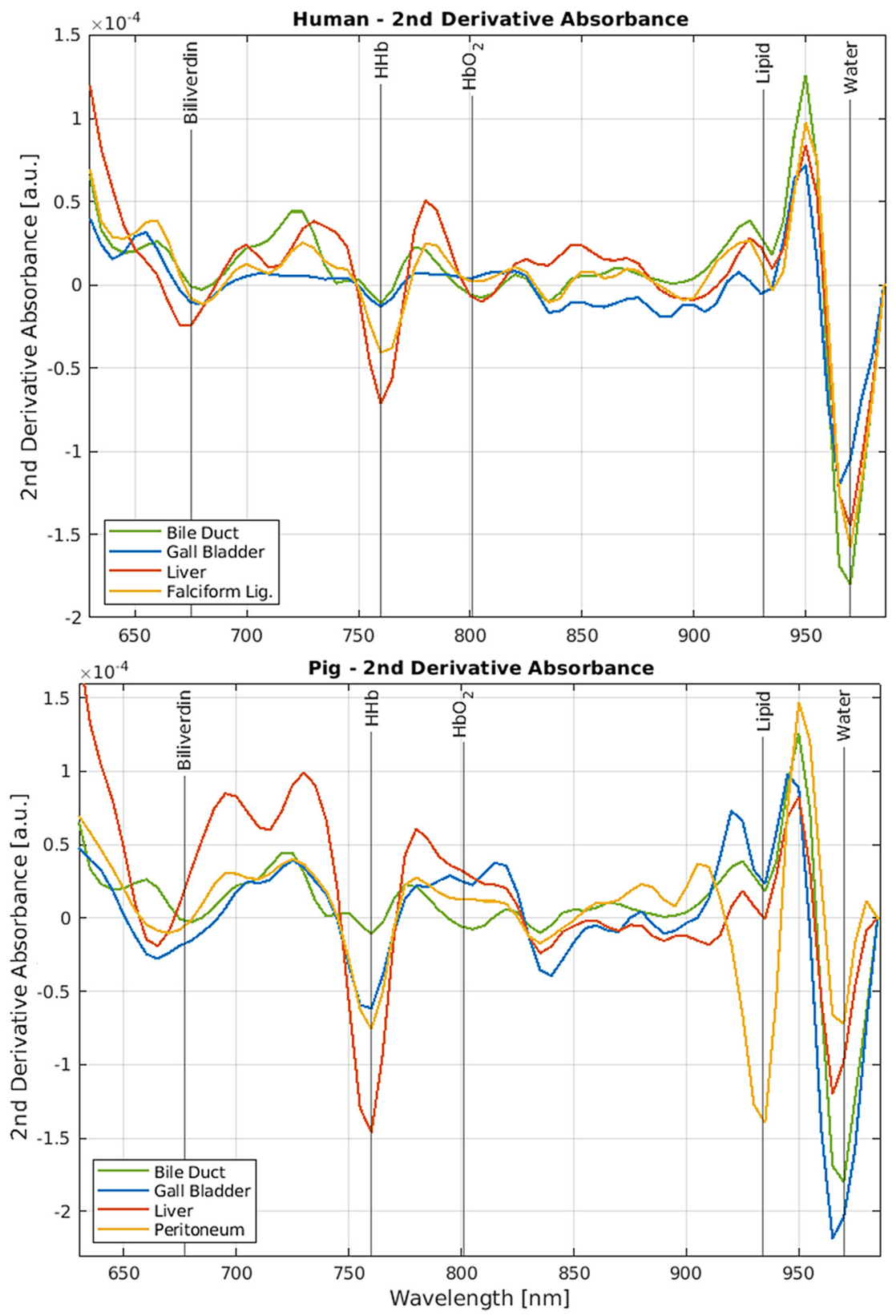

Figure 2: Savizky-Golay 2nd derivative absorbance spectra of human (top) and porcine (bottom) tissue. Vertical lines correspond to biliverdin $=675$; deoxyhemoglobin $(\mathrm{HHb})=760$; oxyhemoglobin $\left(\mathrm{HbO}_{2}\right)=800$; lipid $=930$; water $=970 \mathrm{~nm}$.

Given that the liver contains virtually no contractual tissue, it is expected that the spectral contribution of elastin (broad absorption from 550 to $850 \mathrm{~nm}$ ) is minimal. For the gall bladder both collagen and elastin could contribute to the observed broad absorbance, given the presence of contractual smooth muscle for the expulsion of bile.

Biliverdin (ca. $680 \mathrm{~nm}$ ) is evident in both gall bladder and bile duct derivative spectra in both human and pig. It is not obvious if the liver peak at ca. $660 \mathrm{~nm}$ is a result of another conjugated form of biliverdin or another unidentified chromophore. In both pig and human datasets, the liver (red) has the greatest $\mathrm{HHb}$ peak $(760 \mathrm{~nm})$. This is attributed to two thirds of the hepatic blood supply being partially deoxygenated venous blood. The pig peritoneum has the strongest lipid peak $(930 \mathrm{~nm})$ which is expected. However, the high-lipid content of bile is not apparent in gall bladder or bile duct spectra. This could be due to perioperative fasting reducing the food-related lipid in bile.

\section{Conclusions}

HSI in combination with spectral processing has identified the dual contribution of bile and organ tissue composition to the observed spectra of biliary anatomy. The bile pigment biliverdin and structural proteins collagen and 
elastin were identified as contributors to the absorbance spectra of bile ducts and gall bladder. The concentration of observable biliverdin and lipid content of bile is hypothesized to be influenced by fasting routines prior to operation. This, along with deviations from Beer-Lambert linearity due to scattering or aggregation make the quantification of bile in tissue challenging. This also limits the possibility of using bile as a signature for classification algorithms in image-guided surgery.

One approach to solve this issue is to use deeperpenetrating NIR radiation and to characterize the bile spectrum based on water and lipid content. These water-lipid peaks were the basis of Zuzak et al. [15] analysis in which a laparoscopic hyperspectral imaging system (spectral range of 650-1100 nm) with principle component analysis were used to visualize biliary anatomy of swine. This method along with the inclusion of additional identified chromophores such as biliverdin, could be adapted for the use with the TIVITA ${ }^{\circledR}$ system and the current patient dataset as a method to visualize and classify biliary anatomy intra-operatively.

Research funding: The author states no funding involved. Author contribution: All the authors have accepted responsibility for the entire content of this submitted manuscript and approved submission.

Conflict of interest: The hyperspectral camera used in this publication was developed by Diaspective Vision GmbH. G. S. Cooney was an employee of this company prior to paper submission. H. Köhler is an employee of this company. Informed consent: Informed consent was obtained from all individuals included in this study.

Ethical approval: Experimental hyperspectral measurements from patients for the evaluation of new technology have obtained the ethics approval by the Ethics committee of the University Leipzig under 026/18-ek. The study was conducted according to the Helsinki Declaration.

\section{References}

1. Bai L, Jin C, Wang S. Diagnosis, treatment, and prevention of iatrogenic injury in the lower part of common bile duct. Gastroenterol Hepatol Lett 2019;1:6-10.
2. Stewart L, Way LW. The prevention of laparoscopic bile duct injuries: an analysis of 300 cases of from a human factors and cognitive psychology perspective. Proc Hum Factors Ergon Soc 2007;2:617-20.

3. Ishizawa T, Bandai Y, Kokudo N. Fluorescent cholangiography using indocyanine green for laparoscopic cholecystectomy: an initial experience. Arch Surg 2009;144:381-2.

4. Lu G, Fei B. Medical hyperspectral imaging: a review. J Biomed Optic 2014;19:010901.

5. Jansen-Winkeln B, Holfert N, Köhler H, Moulla Y, Takoh JP, Rabe $\mathrm{SM}$, et al. Determination of the transection margin during colorectal resection with hyperspectral imaging (HSI). Int J Colorectal Dis 2019;34:731-9.

6. Jansen-winkeln B, Maktabi M, Takoh JP, Rabe SM, Barberio M, Köhler $\mathrm{H}$, et al. Hyperspectral imaging in gastrointestinal anastomoses. Surgery 2018;89:717-25.

7. Sucher R, Athanasios A, Köhler H, Wagner T, Brunotte M, Lederer A, et al. Hyperspectral Imaging (HSI) in anatomic left liver resection. Int J Surg Case Rep 2019;62:108-11.

8. Urade T, Felli E, Barberio M, Al-Taher M, Felli E, Goffin L, et al. Hyperspectral enhanced reality (HYPER) for anatomical; liver resection. Surg Endosc 2020;34:1745-53.

9. Köhler H, Jansen-Winkeln B, Maktabi M, Barberio M, Takoh J, Holfert $\mathrm{N}$, et al. Evaluation of hyperspectral imaging (HSI) for the measurement of ischemic conditioning effects of the gastric conduit during esophagectomy. Surg Endosc 2019;33:3775-82.

10. Boyer JL. Bile formation and secretion. Compr Physiol 2013;3: 1035-78.

11. Goncharova I, Jašprová J, Vítek L, Urbanová M. Photoisomerization and oxidation of bilirubin in mammals is dependent on albumin binding. Anal Biochem 2015;490:34-45.

12. Nachabé R, Evers DJ, Hendriks BH, Lucassen GW, van der Voort $M$, Wesseling J, et al. Effect of bile absorption coefficients on the estimation of liver tissue optical properties and related implications in discriminating healthy and tumorous samples. Biomed Optic Express 2011;2:600-14.

13. Chapman GB, Eagles DA. Ultrastructural features of Glisson's capsule and the overlying mesothelium in rat, monkey, and pike liver. Tissue Cell 2007;39:343-51.

14. Konugolu S, Sekar V, Mora AD. Diffuse optical characterization of collagen absorption from 500 to $1700 \mathrm{~nm}$. J Biomed Optic 2017; 22:015006.

15. Zuzak KJ, Naik SC, Alexandrakis G, Hawkins D, Behbehani K, Livingston $\mathrm{E}$. Intraoperative bile duct visualization using nearinfrared hyperspectral video imaging. Am J Surg 2008;195:491-7.

16. Sutor DJ, Wilkie LI. Diurnal variations in the $\mathrm{pH}$ of pathological gallbladder bile. Gut 1976;17:971-4.

17. Hofmann AF, Mysels KJ. Bile acid solubility and precipitation in vitro and in vivo: the role of conjugation, $\mathrm{pH}$, and $\mathrm{Ca}^{2+}$ ions. J Lipid Res 1992;33:617-26. 